\title{
Mathematical morphology approach for single-channel processing of auscultative sound
}

\author{
Alexandr G. Rudnitskii \\ Institute of Hydromechanics, National Academy of Sciences of Ukraine, \\ ul. Zhelyaebova 8/4, Kyiv, 03680 Ukraine
}

How to cite this paper: Rudnitskii A. G. (2019). Mathematical morphology approach for single-channel processing of auscultative sound. Journal of Applied Mathematics and Computation, 3(5), 616-626.

DOI: 10.26855/jamc.2019.09.001

*Corresponding author: Alexandr G. Rudnitskii, Institute of Hydromechanics, National Academy of Sciences of Ukraine, ul. Zhelyaebova 8/4, Kyiv, 03680 Ukraine.

Email: rudnitskii@mail.ru

\begin{abstract}
The problem of isolating breath and cardiac sounds in the total signal registered on a human thorax has been of interest to physicians and researchers in the last two decades. Many algorithms have been developed to address this issue. In this paper we propose a new technique for isolating respiratory sound from a combined signal registered on the chest wall. The method is based on a hybrid algorithm using a mathematical morphological approach and spectral subtraction technique. This approach has been tested both on the artificially simulated data and on real auscultative sounds. Evaluation of efficiency by means of auditory, visual and numerical analysis shown that the proposed strategy is a promising alternative to existing technologies of separation of auscultative signals on its natural components.
\end{abstract}

\section{Keywords}

mathematical morphology, spectral subtraction, digital auscultation, noise suppression, respiratory sound analysis

\section{Introduction}

Listening to the sounds at the human chest wall for diagnosing of the heart, lungs and other organs has been a well known method from the early stages of medicine. Due to its non invasive nature, availability and simplicity this method remains one of the most common ways used by physicians before more costly complex and modern medical examinations and tests. Of course, new approaches can allow get more accurate information of diseases than auscultation $^{1}$, but they require expensive equipment and specialised personnel.

Moreover, a number of diseases cause differentiations of auscultative signs in earlier stages, before they can be detected in other advanced imaging approaches (e.g., heart murmurs and abnormal heart sounds reasoned in structural defects and abnormalities in heart valves). Furthermore, auscultation is an environmentally friendly diagnostic method that allows to decrease the radiation load on the patient during CT-diagnosis and thereby reduce the risk of cancer [1].

The broad opportunities that can be afforded by computer processing create a new region of auscultation such as digital auscultation with "smart" electronic stethoscopes and diagnostic computer complexes.

There are many challenges to developing such devices, including determining an appropriate type of sensor (accelerometers or pressure sensors), designing an effective attachment method and so on. This paper focuses on removing (reducing) noise in useful signal registered on human chest wall. Presence of noise in auscultative signals is a major problem which is inevitable in practice. Noise contained in the signal is introduced by a variety of sources such as ambient noise (e.g. background noise and stomach rumbling), thoracic muscular noise, peristaltic intestine noise,

\footnotetext{
${ }^{1}$ Auscultation is listening and interpretation to breath, heart or abdominal sounds at body surface through a stethoscope
} 
fetal sounds (if the subject is pregnant), non-optimal recording sites, electronic noise and so on. If these noises are not removed, the accuracy of the diagnostic features will be seriously affected and ultimately influence the diagnostic system's performance.

So, in order to realize appealing advantages of computer-aided auscultation, common signal registered on thorax should be separated into respiratory and cardiac parts. Given that both heart sounds and respiratory noises are non-stationary, non-gaussian, and there exists an overlapping of these signals in time and frequency domains this problem is rather difficult. To date, a number of approaches have been reported in the literature for solving such a difficult task.

First, one can use a breath-holding signal. Unfortunately, this simple approach cannot be used for certain categories of patients and can be the cause of changes in the characteristics of the heart signal.

Methods are also used where the influence of cardiac noise and noise related to muscle vibration in the frequency region lower than $70-100 \mathrm{~Hz}$ is suppressed by bandpass filters, which suppress the low-frequency range of the auscultative signal [2]. However, such a head-on simple method can result in loss of important diagnostic signal information [3].

If it is desired that the low-frequency content of the spectra be retained, more refined methods are used: a cutting out of segments containing heard sounds followed by interpolation of the missing data, the use of multichannel and adaptive filtering techniques, wavelet analysis or blind source separation [4-16]. All of these methods reduce interference, but signal distortion is still audible.

In this work, in order to solve the problem of isolating two components (cardiac sounds and the remainder, the sum of respiratory and background noise) in a signal recorded on the chest wall, it is proposed to use the hybrid method based on well known Spectral subtraction (SS) technique and recently highly recommended approach based on Mathematical Morphology (MM) [17,18].

Spectral subtraction is a single channel noise reduction algorithm for signal enhancement [19]. The basic steps of the algorithm are: transformation signal from time to frequency domain; noise spectrum estimation; subtraction noise spectrum from the spectrum of initial noisy signal; resynthesizing the enhanced signal. Crucial problem of this approach is the problem of noise spectrum estimation because complex and nonstationary nature of thorax sounds.

Mathematical Morphology is a theory based on concepts of topology, geometry and set theory. It focuses on analysis of the shapes, extracts of geometrical information from an unknown objects (image) and to date it is a powerful tool for image analysis and enhancement.

This paper is organized as follows. First, the brief consideration of the MM and SS methods are given. Further, the paper describes the results of applying the method to real-life sound signals recorded on the human chest wall. The paper concludes with a discussion of the results and possibilities of the method.

\section{Methods}

\subsection{Short-time Spectral Method}

Short-time Spectral Method is based on short-time Fourier transform (STFT). This transform is appropriate for nonstationary signal. Firstly the analysed signal is subdivided into a number of successive short segments where it is assumed that each segment is stationary. Then the sequence of short sub-recordings of the signal is converted to the frequency domain.

The continuous time-frequency representation of a signal $x(t)$ is shown as follows

$$
X(t, \omega)=\int_{-\infty}^{\infty} x(\tau) w(t-\tau) e^{-j \omega \tau} d \tau
$$

where $w(t)$ is the analysis window and $\mathrm{t}$ is time. The polar form of this transformation can be also written as

$$
X(t, \omega)=|X(t, \omega)| e^{-j \Psi(t, \omega)},
$$

where $|X(t, \omega)|$ and $\Psi(t, \omega)$ are short-time magnitude and phase spectrum respectively. 
Short-time Spectral Method for noise reduction is based on the assumption that spectrums of additive independent signals are also additive and that this property is approximately true for short-time estimate. In frequency domain, the noise spectrum estimation is removed from the noisy spectrum, leaving an approximation of the spectrum of the clean signal. Then the estimation of clean signal spectrum is converted to the time domain using an inverse Short-time Fourier transform.

Let $s(n)$ and $d(n)$ be represented by a windowed clean signal and noise signal respectively. The sum of the two is then denoted by $x(n)$ (registered signal),

$$
x(n)=s(n)+d(n) .
$$

In a frequency domain (3) can be expressed as

$$
X(k)=S(k)+D(k),
$$

where $X(k), S(k)$ and $D(k)$ are Fourier transforms of noisy signal, clean signal and noise in a segment, respectively.

To obtain the short-term power spectrum, $X(k)$ in the above equation has to be multiplied by its conjugate $X^{*}(k)$. Then

$$
|X(k)|^{2}=|S(k)|^{2}+|D(k)|^{2}+S^{*}(k) \cdot D(k)+S(k) \cdot D^{*}(k),
$$

or, in polar form

$$
|X(k)|^{2}=|S(k)|^{2}+|D(k)|^{2}+|D(k)||S(k)|\left(e^{-i \theta_{S}} e^{i \theta_{D}}+e^{-i \theta_{D}} e^{i \theta_{S}}\right),
$$

where $\theta_{S}(k)$ is the short-time phase spectrum of clean signal in a frame and $\theta_{D}(k)$ is the short-time phase spectrum of noise.

Cross terms and $|D(k)|^{2}$ in the above equation usually are approximated as $E\left\{|D(k)|^{2}\right\}, E\left\{S^{*}(k) \cdot D(k)\right\}$ and $E\{S(k)$. $\left.D^{*}(k)\right\}$, where $E\{\cdot\}$ denotes the expectation operator. If noise has zero mean and clean signal and noise are assumed to be uncorrelated, then the cross terms reduce to zero and an estimate of the clean signal power spectrum $|\hat{S}(k)|^{2}$ can be obtained by the following expression:

$$
|\hat{S}(k)|^{2}=|X(k)|^{2}-|\hat{D}(k)|^{2},
$$

where $|\hat{D}(k)|^{2}$ is the estimation of noise power spectrum.

The estimation of enhanced complex spectrum can be obtained by using modified magnitude spectrum and unchanged phase spectrum of the noisy signal

$$
\hat{S}(k)=\left(|X(k)|^{2}-|\hat{D}(k)|^{2}\right)^{1 / 2} e^{j \theta_{x}(k)}
$$

and enhanced signal frame is synthesized by performing the inverse FT as

$$
\hat{s}(n)=\operatorname{Re}\{\operatorname{IFFT}[\hat{S}(k)]\},
$$

where $s(n)$ represents the enhanced signal frame and $\operatorname{Re}(\cdot)$ denotes the real part of the number inside. The final signal is reconstructed by using the overlap and add method.

This approach does not need any assumptions concerning the signal and it is quite simple computationally. However, crucial problem of this method is an estimate of the noise. There are many methods of noise estimation, of which ones are more suitable to some type of situations and others, for different ones.

One of the new methods is proposed and examined in the following sections.

\subsection{Mathematical morphology}

Mathematical morphology is a branch of mathematics which stems from works of J.Serra and G. Matheron [17, 18]. This theory encompasses many various mathematical disciplines from topology, discrete and continuous geometry, set theory, random functions and lattice theory. MM analyzes spatial structures, shapes and the forms of the objects. This method has been mainly used to analyze images since its launch. Technique of processing consists in comparing the 
objects to be analyzed (image) with another object of known form, called structuring element. It may have different shapes and sizes (disk or linear for example). The choice of structuring element depends on analyzed image and is crucial to the success of the handling.

Initially MM dealt with binary images only (black and white or two tone images). Then binary morphology was extended to grayscale images as well.

The basic operations were dilation and erosion, defined in terms of Minkowski addition and subtraction [20].

A grayscale image $f$ is a mapping of a subset $D_{f}$ of $Z^{n}$, called the definition domain of $f$, into a bounded set of nonnegative integers $N_{0}$,

$$
f: D_{f} \subset Z^{n} \rightarrow\left\{0,1, \ldots, t_{\max }\right\},
$$

where $t_{\max }$ is the maximum value of the data type used, (e.g., 255 in a gray scale 8-bit image).

The grayscale erosion of a set $X$ by a structuring element (SE) $B$ is defined as follows:

$$
\left[\varepsilon_{B}(f)\right](x)=\min _{b \in B} f(x+b) .
$$

The grayscale dilation $\delta_{B}(X)$ of a set $X$ by a structuring element $B$ is expressed as below:

$$
\left[\delta_{B}(f)\right](x)=\max _{b \in B} f(x+b) .
$$

The combination of dilation and erosion produces opening and closing operations.

The opening $\gamma_{B}(f)$ of an image $f$ by a structuring element $B$ is dilation of eroded image in order to recover this image as it is possible:

$$
\gamma_{B}(f)=\delta_{\hat{B}}\left[\varepsilon_{B}(f)\right] .
$$

In contrast, the idea behind closing of an image $f$ by a structuring element $B$ is to erode a dilated image for its recover

$$
\varphi_{B}(f)=\varepsilon_{B}\left[\delta_{B}(f)\right] .
$$

The basic morphological filters are the morphological opening and closing [21].

Gray scale opening may be used to reduce high intensity values of thin areas which can not adjust to the SE, such that these regions merge with their background. On the same lines, gray scale closing may be used to increase low intensity values of thin regions to level them with their background.

It is important to note that since the operations of closing and opening do not commute, in general a different result is expected when the order of closing and opening operations is switched.

\section{Processing}

The proposed algorithm has been applied on both artificial simulated signals and real auscultative signals registered on human chest wall.

\subsection{Testing of artificial signal}

Artificial signal $x(t)$ was modeled as sum of three components - cardiac sounds $h(t)$, respiration sounds $b(t)$ and background noise $d(t)$ :

$$
x(t)=\alpha h(t)+\beta b(t)+\gamma d(t),
$$

where the values of $\alpha, \beta$ and $\gamma$ were determined by the desired level of the corresponding components and $t$ is time. The signals for cardiac sounds were taken from the R.A.L.E. database on the Internet [22]. Breathing sounds were modeled as amplitude modulated random signal with a power law spectrum. For imitation of noisy environment was generated a stationary colored noise. All these components were normalized (zero for the mean and 1 for the standard deviation). Power-law spectrum for respiratory signals was chosen because such spectrums were observed in experiments for vesicular breathing noises in wide frequency range [23].

One of such synthesized signals is shown on Fig 1. For this case $\alpha=1, \beta=0.3$ and $\gamma=0.15$. 


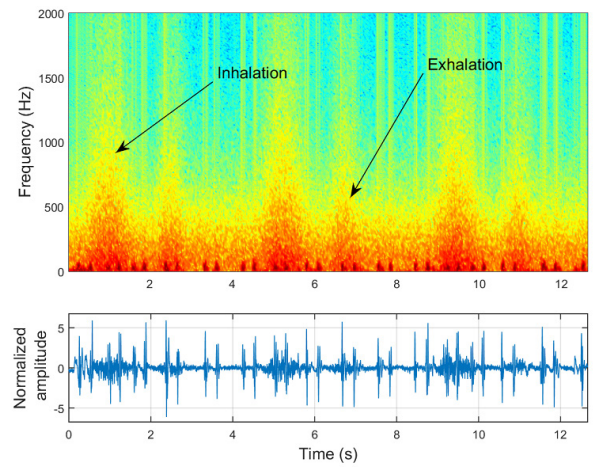

Figure 1. Artificially synthesized signal. Top panel - spectrogram of the simulated signal, bottom panel - synthesized signal in time domain.

The spectrogram of the signals was calculated by applying the discrete STFT to every 100 ms segment of data with a $25 \%$ overlap between adjacent segments using a Hanning window to avoid end-point discontinuity. Fig 1 shows one of synthesized signals in time domain (bottom panel), and spectrogram of the signal (top panel).

For comparing Fig 2 presents the typical real-life auscultative signal recorded at $1 \mathrm{~L} / \mathrm{s}$ flow rate in time-frequency domain (top panel) and in time domain (bottom panel).

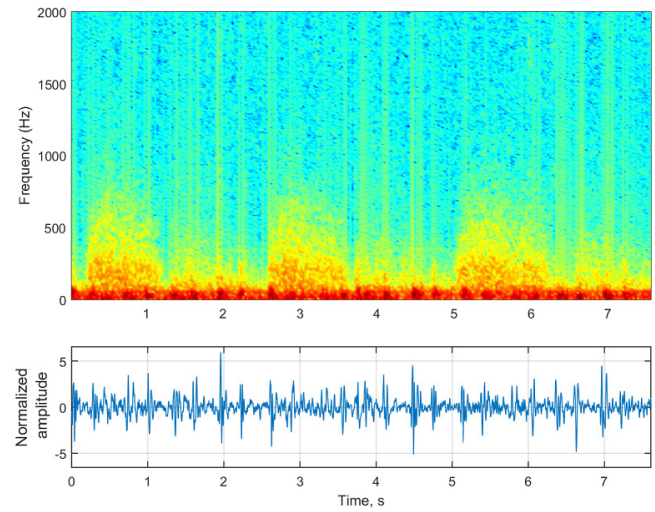

Figure 2. The real-life auscultative signal at flow rate $1 \mathrm{~L} / \mathrm{min}$ : top panel - spectrogram of signal; bottom panel - signal in time domain.

The horizontal axis is the duration of the recording in seconds and the vertical axis on the spectrogram is the frequency range. The magnitude of the power spectrum is shown by color, where the red color represents high intensity of signal whereas the blue represents low intensity. As it can be noticed, the inhalation segments of the signal have much higher frequency components than exhalation segments. So, inhalation sounds are louder than exhalation sounds over the chest wall and this observation is fairly consistent among the subjects in real-life experiments.

The spectrogram of the recorded auscultative sound can be thought as an image with a number of gray levels. This image has respiratory sounds as background and heart sounds (HS) as vertical lines on this background. The line type feature of segments that include HS is not changed while the background textures of the image are different (this HS structures always have the shape of vertical lines and background changes during inhale and exhale).

This leads to an approach for detecting of segments that include HS based on MM methodology, because mathematical morphology is particularly effective for processing images features shape of which are known a priori. Since the HS is known to be vertical lines on spectrogram, an algorithm of MM filtration with linear structuring element has to robustly extract HS segments from the original spectrogram.

It is well known that the combination close-opening morphological operation leads to larger amplitude than the original signal while the result of open-closing operation has lower amplitude. The combination of these operations gives more precise extraction of spikes: 


$$
g=\min \left(\left\{\gamma_{B}\left[\varphi_{B}(\mathrm{f})\right]+\varphi_{B}\left[\gamma_{B}(f)\right]\right\} / 2, f\right),
$$

where $B$ is linear structuring element with width 1 and length $L$. This filter removes linear shapes when the shape and the structuring element are approximately orthogonal and preserves linear shapes when the structuring element and the shape are about parallel. It gives a possibility to remove the linear shapes from an image and to replace them with their (local) background. Thus, the background of the image can be obtained by morphological filtering using a linear structuring element that is orthogonal to the ordinate axis.

To satisfy the extracting of HS segments, the maximum length of structuring elements was selected close to the 1.5 length of HS segments (about $0.35 \mathrm{sec}$ for all images). It is important, that our filter, which removes HS segments, consists of a sequence of operations of morphological opening and closing with increasing length of the structuring elements. Minimum length of a structural element is about $0.0172 \mathrm{~s}$, maximum length is $0.3 \mathrm{~s}$ with a step of $0.0086 \mathrm{~s}$. This provided a smoother and better removal of HS segments from the original spectrogram.

The results of morphological filtration of the spectrogram of simulated signal (Fig 1) are shown on Fig 3. Spectrogram without HS segments is displayed on the top panel and spectrogram of HS on flat background is presented on the bottom panel.

As mentioned before, accurate estimation of noise is crucial to any successful signal enhancement system and now after getting of spectrograms of cardiac and respiratory signals, spectral subtraction method (4)-(5) can be implemented for separating of heart and respiratory sounds.

Fig 4 illustrates an example for artificial signal in time domain, separated by the algorithm used in this work. Zoomed fragments of the original noisy signal and related superimposed breathing sound, and heart sound signal are shown on the middle and bottom sub-figures respectively. On this figure green line describes the initial signal including the breath and cardiac sounds and the stationary background noise, red line is heart signal and blue line is respiratory signal.

The visual evaluation performance of algorithm in time-frequency domain may be done in Fig 5. It is apparent that original signal decomposed on HS and respiratory sound almost without degradation of components.
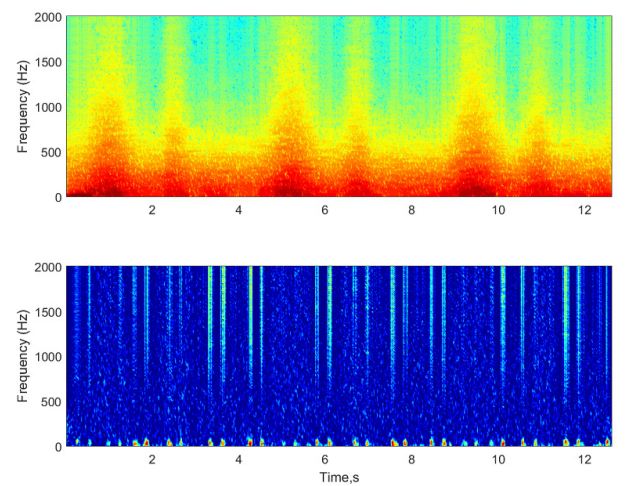

Figure 3. Spectrograms of the artificial lung sound signal after MM filtering: top panel - filtered image; bottom panel - HS segments.

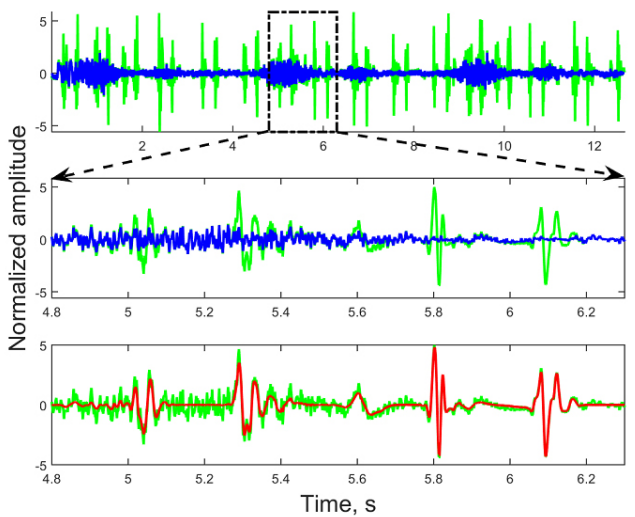

Figure 4. The signals separated with the proposed algorithm for artificial signal. Green line is original noisy signal, red line is heart signal and blue line is respiratory signal. 
For quantitative evaluation of the performance of algorithm vectors divergence angle $\varphi=\arccos \{\cos [x(t), y(t)]\}$ was used. This measure has a linear character of the change in its values from $0^{\circ}$ when there is complete coincidence of functions $x(t)$ and $y(t)$ up to $90^{\circ}$ for their complete orthogonality (zero correlation).
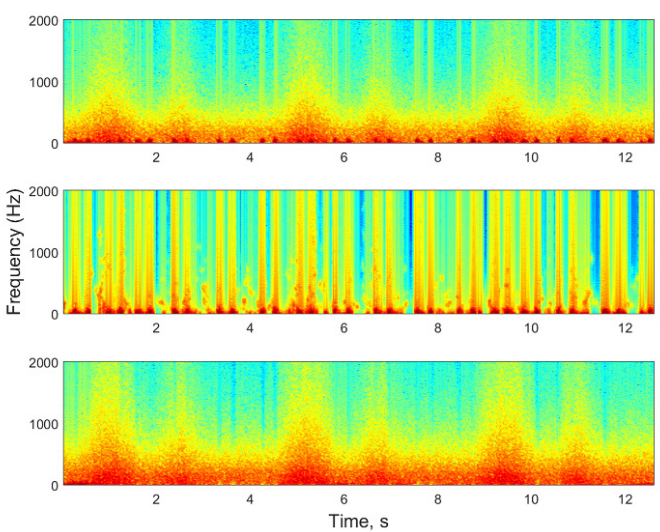

Figure 5. Spectrograms of the artificial signal: top panel - original noised signal; middle panel - spectrogram of heart sound estimation; bottom panel - spectrogram of breathing sound estimation.

There were evaluated angles between clean cardiac signal $\alpha h(\mathrm{t})$ and its estimation $\alpha \hat{h}(\mathrm{t}) \varphi_{0}=\arccos \{\cos [\alpha h(\mathrm{t}), \alpha \hat{h}(\mathrm{t})]\}$ and between difference $x(\mathrm{t})-\alpha \hat{h}(\mathrm{t})$ and estimation of cardiac signal $\varphi_{1}=\arccos \{\cos [x(\mathrm{t})-\alpha \hat{h}(\mathrm{t}), \alpha \hat{h}(\mathrm{t})]\}$. Last evaluation for $\varphi_{1}$ was done because in real-life case a clean signal is unknown, but since cardiac sounds are assumed to be uncorrelated with sum of respiration and background noise, the angle between them should be close to $90^{\circ}$.

The estimates of vectors divergence angle were obtained by averaging over 20 realizations of the artificial respiration and noise and they are $\varphi_{0}=12.5 \pm 0.26$ and $\varphi_{1}=79.82 \pm 0.19$ (parameters of simulated signals: $\alpha=1, \beta=0.3$ and $\gamma=0.15$ ).

\subsection{Testing of real-life signals}

The real-life signals subjected to the proposed processing were got by the experimental technique described in [22]. Two-channel registrations of auscultative signals was carried out by sensitive contact piezoelectric accelerometers. Sensors were placed on the second intercostal space anteriorly (midclavicular line) symmetrically on the left and right upper lung lobes. The subjects were asked to maintain their breath at 0.5 and $1 \mathrm{~L} / \mathrm{s}$ flow rates during $4-5$ breathing cycles at each desired flow. Auscultative records of 16 healthy volunteer subjects aged 10-26 years ( 3 females and 13 males) ere made. The recorded signals were amplified, band pass filtered in the range of 7-4,000 $\mathrm{Hz}$ and digitized at 8,000 $\mathrm{Hz}$ sampling rate using 12 bits per sample.

Typical spectrogram of auscultative signal registered at $1 \mathrm{~L} / \mathrm{s}$ is shown on Fig 2 while Fig 6 displays the result of morphological filtering of this spectrogram.
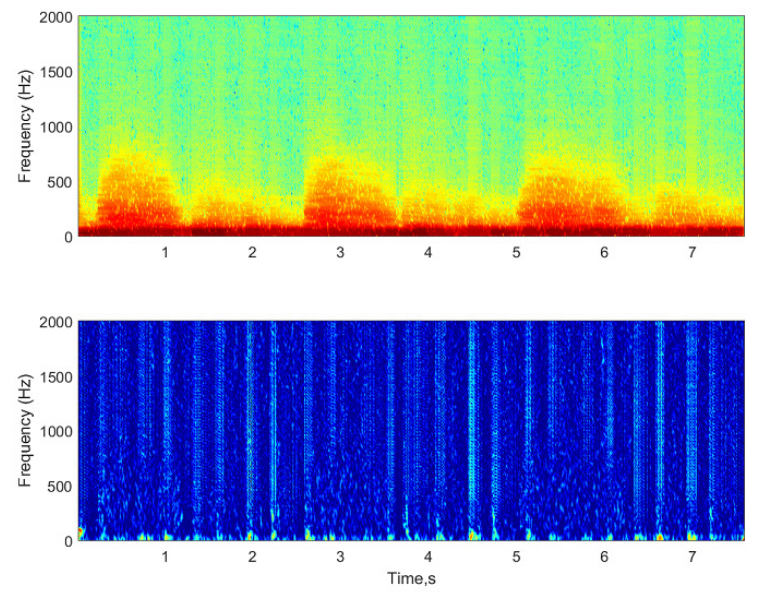

Figure 6. Spectrograms of the lung sound signal after MM filtering: top pane - filtered image; bottom panel - HS segments. 
Spectrogram without HS segments is shown on the top panel and image of HS on flat background is presented on the bottom panel.

Fig 7 illustrates the result of the spectral subtraction method with noise estimation performed by morphological operations. Zoomed fragment contains a pause (silence) between breathing cycles, inhalation and heart sounds. It can be seen that the segments of signal between cardiac sounds remain unchanged while fragments of heart sound in these fields are clean from noise.

The visual evaluation performance of algorithm in time-frequency domain may be seen on Fig 8 .

When respiratory intensity is low, the respiratory noise may be difficult for listening on the background of ambient noise and heart sounds. Nevertheless, in this case, the proposed algorithm also demonstrates good quality of dividing the total signal into its natural components - breath sounds and heart sounds. This situation is shown on Figs 9-10.

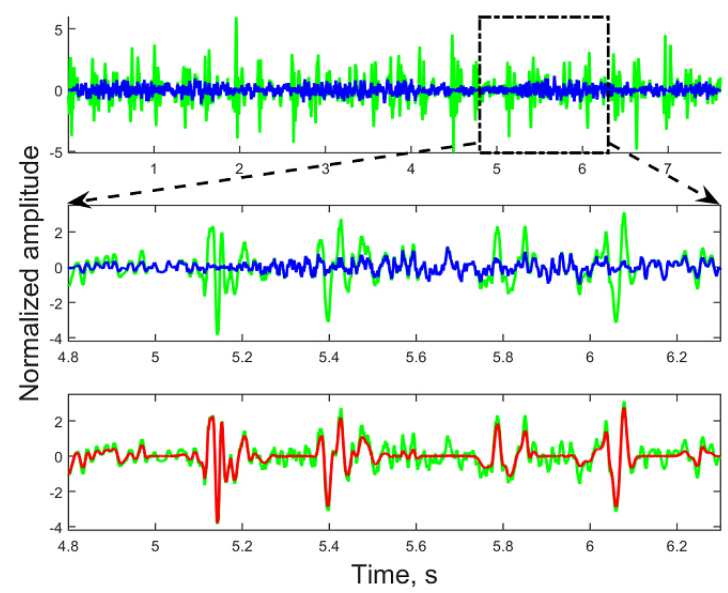

Figure 7. The signals separated with the proposed algorithm at flow rate $1 \mathrm{~L} / \mathrm{min}$. Green line is original noisy signal, red line is estimation of heart signal and blue line is respiratory signal.
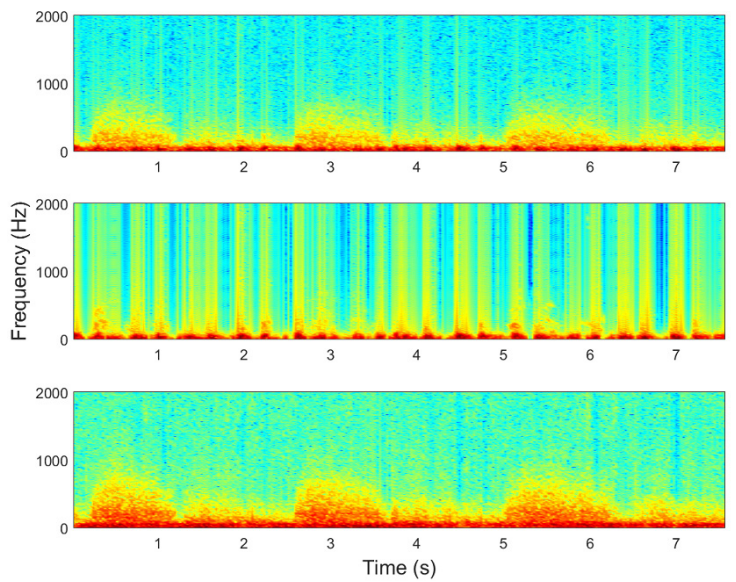

Figure 8. Spectrograms of the lungs sound signal at flow rate $1 \mathrm{~L} / \mathrm{min}$ : top panel - original noised signal; middle panel - spectrogram of estimated heart sound; bottom panel is spectrogram of breathing sound.

Since in real situation clean signal is unknown, quantitative evaluation of the performance of the algorithm was done for vectors divergence angle between the estimation of heart sounds and the residue (respiration + background noise). The angle between them should be close to $90^{\circ}$ because we assume that they are uncorrelated. In our case, $\varphi \approx 75^{\circ}$ when the intensity of breathing was about $0.5 \mathrm{~L} / \mathrm{min}$ and $\varphi \approx 70^{\circ}$ at flow rate $1 \mathrm{~L} / \mathrm{min}$. As one can see angle $\varphi$ takes values sufficiently close to $90^{\circ}$ for both regimes of breathing. Vectors divergence angle $\varphi$ isn't exactly $90^{\circ}$ because heart and respiratory signals are not exactly linearly mixed but are convolved due to transmission through fat, skin and lung tissues. 


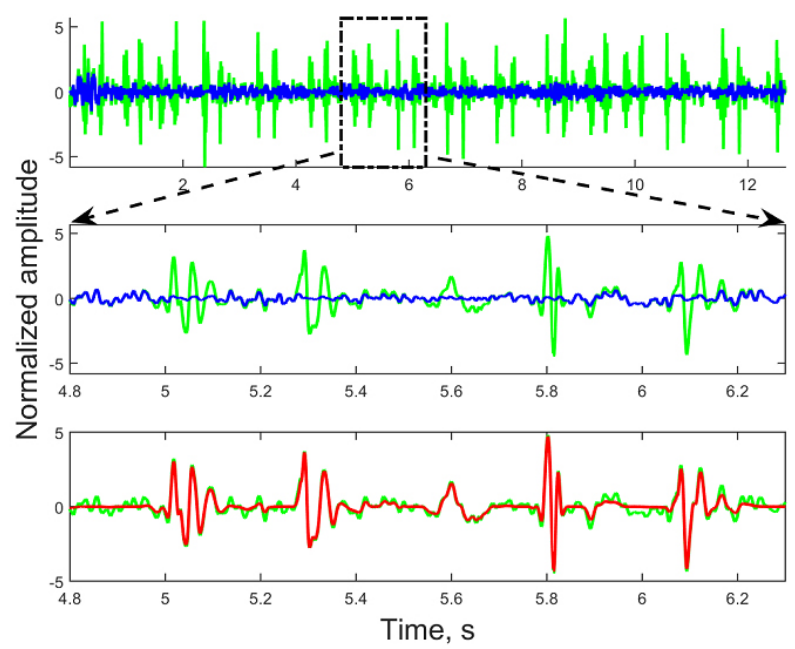

Figure 9. The real-life signals separated with the proposed algorithm at flow rate $0.5 \mathrm{~L} / \mathrm{min}$. Green line - original noisy signal, red line is an estimate of heart signal and blue line is filtered respiratory signal.
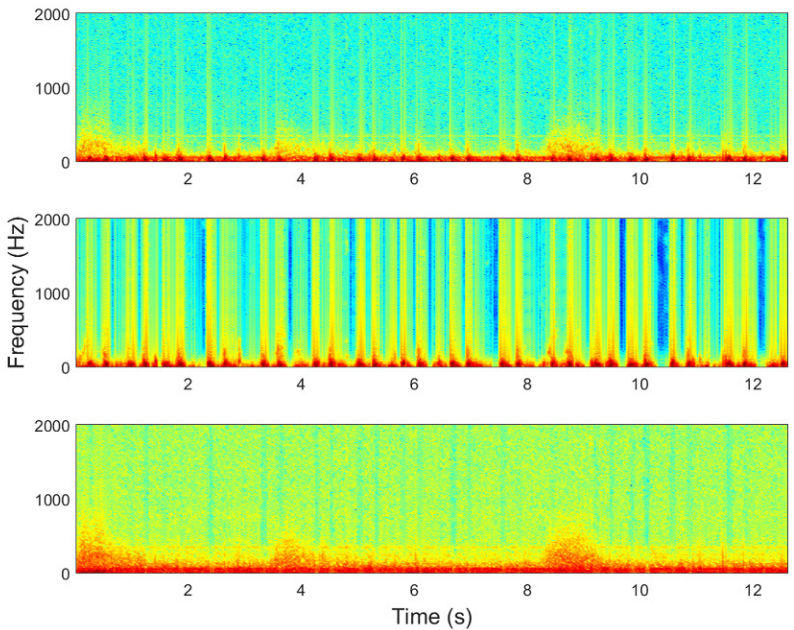

Figure 10. Spectrograms of the lung sound signal at flow rate $0.5 \mathrm{~L} / \mathrm{min}$ : top panel - original noised signal; middle panel spectrogram of estimated heart sound; bottom panel - spectrogram of filtered breathing sound.

\section{Conclusions}

In this investigation, we proposed a hybrid procedure to separate the auscultative signal into its natural components, breath and heart sounds. Mathematical morphology approach proposed in our work can divide spectrogram of registered signal into foreground and background. Foreground includes heart sound segments of registered auscultative signal while background is a sum of ambient noise and respiratory sounds. It is the key to separate the respiratory noise and cardiac sound in the auscultative signal. After this we successfully separate the heart and breathing sound by spectral subtraction methodology.

In order to assess the performance of the proposed processing technique we used two approaches, subjective and objective.

Subjective visual evaluation of performance of proposed method was carried out in terms of both spectrogram representations and in time domain. This visual inspection revealed rather well results for both artificial and real-life signals. The second tool we used for subjective evaluation of the proposed approach was listening of real-life signals. These tests were performed by skilled physicians. This evaluation also confirmed the effectiveness of the proposed 
technique in terms of noise removal and preservation of the features of the signal of interest.

The quantitative analysis of the signals has shown that the introduced approach allows effectively separate the auscultative sounds on its natural components.

In our work it was shown that on the basis of combination of MM and SS algorithms it is possible to observe a large amount of information hidden and disguised in signal registered on the human thorax.

There are several areas that require further research to make our approach more effective. Firstly, it is the work to optimize the algorithm in terms of its performance, and secondly, it is the change of some details of the algorithm for processing signals recorded in patients with lung and heart diseases (it is the main aim). Also as future work we plan to test the proposed method in different experimental conditions of real public hospitals.

\section{References}

[1] Savage N. (2010) Medical imagers lower the dose. IEEE Spectr., 47, N. 3, 14-15.

[2] Charbonneau G., Raccineux J.L., Sudraud M., and Tuchais E. (1983) An accurate recording system and its use in breath sounds spectral analysis J. Appl. Physiol. 55, 1120.

[3] Gavriely N., Palti Y., and Alroy G. (1981) Spectral characteristics of normal breath sounds. J. Appl. Physiol. $50,307$.

[4] Iyer V.K., Ramamoorthy P.A., Fan H. and Ploysongsang Y. (1986) Reduction of heart sounds from lung sounds by adaptive filtering. J. IEEE Trans. Biomed. Eng., 33, N. 12, 1141-1148.

[5] Hadjileontiadis L.J., Panas S.M. (1998) A wavelet based reduction of heart sounds noise from lung sounds, International Journal of Medical Informatics 52 (October-December), 183-190.

[6] Tinati M.A., Bouzerdoum A., Mazumdar J. (1996) Modified adaptive line enhancement filter and its application to heart sound noise cancellation, in: Proceeding of the International Symposium on Signal Processing and its Applications (ISSPA), 2, 815-818.

[7] Charleston S., Azimi-Sadjadi M.R. (1997) Reduced order Kalman filtering for the enhancement of respiratory sounds. IEEE Transactions on Biomedical Engineering 44, October (10), 1006-1019.

[8] Hadjileontiadis L.J., Panas S.M. (1998) A wavelet based reduction of heart sounds noise from lung sounds, International Journal of Medical Informatics, 52 (October-December), 183-190.

[9] Ergen B., Tatar V. (2001) The analysis of heart sounds based on linear and high order statistical methods, in: Proceedings of the 23rd Annual International Conference of the IEEE-Engineering in Medicine and Biology Society, 2139-2141.

[10] Rudnitskii A.G. (2001) Two-Channel Processing of Signals for the Separation of Breath and Cardiac Sounds Acoustical Physics, 47, N. 3, 353-360.

[11] Messer S.R., Agzarian J., Abbott D. (2001) Optimal wavelet denoising for phonocardiograms. Microelectronics Journal, 32, December (12), 931-941.

[12] Pourazad M., Moussavi Z., Farahmand F., Ward R. (2005) Heart sounds separation from lung sounds using independent component analysis, in: Proceedings of the Conference of the IEEE-Engineering in Medicine and Biology Society, 3, 2736-2739.

[13] Flores-Tapia D., Moussavi Z.M., Thomas G. (2007) Heart sound cancellation based on multiscale products and linear prediction. IEEE Transactions on Biomedical Engineering, 54, February (2), 234-243.

[14] Rudnitskii A.G. (2014) Using Nonlocal Means to Separate Cardiac and Respiration Sounds. Acoustical Physics, 60, N. 6, 719-726.

[15] Rudnitskii A.G. (2015) Heart sound cancellation from lung sound recordings using empirical mode decomposition technique. Proceedings of the 40th International Lung Sounds Association Conference, 58-59.

[16] Gnitecki J. and Moussavi Z. (2007) Separating heart sounds from lung sounds. IEEE Eng. Med. Biol. Mag. 26, $20-29$. 
[17] Matheron G. (1975) Random sets and integral geometry. John Wiley \& Sons, New York,.

[18] Haralick, R.M., Sternberg, S.R., Zhuang, X. (1987) Image analysis using mathematical morphology. IEEE Trans. PAMI, 9, N. 4, 532-550.

[19] Boll S. (1979) Suppression of acoustic noise in speech using spectral subtraction. IEEE Trans. Acoust. Speech, Signal Process., 27, 113-120.

[20] Minkowski H., (1903) Volumen und Oberfläche. Math. Ann., 57, 447-495.

[21] Heijmans, H. J. A. M. (1994). Morphological image operators. Advances in electronics and electron physics, supplement. Academic Press, Boston, MA.

[22] "R.a.l.e. repository" [online], http://www.rale.ca/Recordings.htm.

[23] Gavriely N, Palti Y, Alroy G. (1981) Spectral characteristics of normal breath sounds. J Appl Physiol Respir Environ Exerc Physiol., 50, N. 2, 307-314.

[24] Makarenkov A.P. and Rudnitskii A. G. (1995) Diagnosis of lung pathologies by two-channel processing of breathing sounds. Acoust. Phys., 41, 234-238. 